EDITORIAL

\title{
The role of vasodilators in the prevention and treatment of no-reflow following percutaneous coronary intervention
}

\section{S A Harding}

Heart 2006;92:1191-1193. doi: 10.1136/hrt.2006.088427

The routine use of vasodilators in patients with acute coronary syndromes or other groups undergoing percutaneous coronary intervention ( $\mathrm{PCl}$ ) cannot be recommended at present. However, in the event of noreflow occurring following $\mathrm{PCl}$, intracoronary adenosine or verapamil should be administered

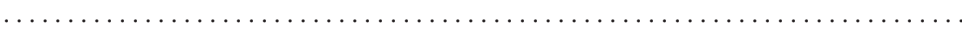

$\mathrm{N}$ o-reflow has been variably defined. In the setting of percutaneous coronary intervention (PCI) it is best defined as inadequate myocardial perfusion in the target vessel territory without evidence of mechanical epicardial vessel obstruction. Angiographic no-reflow defined as less than Thrombolysis in Myocardial Infarction (TIMI) 3 flow occurs in $2 \%$ of all PCIs. ${ }^{1}$ Angiographic no-reflow is associated with an increased incidence of death, myocardial infarction and heart failure. ${ }^{2}$ More recently it has become evident that there is frequently persistent microcirculatory impairment and abnormal tissue level perfusion following PCI despite the presence of TIMI 3 flow (normal epicardial flow). ${ }^{3}$ Microvascular dysfunction is thought to account for $50-70 \%$ of all periprocedural myocyte injury and occurs most frequently in the setting of PCI for acute myocardial infarction or degenerative saphenous vein grafts (SVGs). ${ }^{4}$

Use of a vasodilator to treat no-reflow was first described by Wilson et al in 1989 who reported a favourable response to papaverine in a single patient. ${ }^{5}$ Since this time the effect of several different vasodilators on no-reflow has been investigated. To understand the potential role and limitations of vasodilators in preventing noreflow and periprocedural myocyte injury it is necessary to understand the underlying pathogenesis.

\section{PATHOGENESIS OF NO-REFLOW AND IMPLICATIONS FOR THERAPEUTIC INTERVENTION}

The pathogenesis of the microvascular dysfunction leading to no-reflow is complex. Microvascular spasm, distal embolisation of atheroma and thrombus, microvascular plugging by platelets and leucocytes, endothelial swelling, tissue oedema compressing the microvasculature, oxidative stress and inflammation may all play a role. ${ }^{4}$ The multiple underlying mechanisms contributing to the development of noreflow make it likely that a single therapeutic strategy will be only partially effective in preventing or treating no-reflow. It also important to understand that mechanisms involved in the pathogenesis of no-reflow may vary depending on the clinical scenario. For example, in the setting of acute myocardial infarction, disruption of the microvasculature and the surrounding tissues due to prolonged ischaemia along with reperfusion injury play a prominent role whereas in SVG interventions distal atheroembolism is the predominant mechanism.

This variation in the underlying pathophysiology raises the possibility that a therapeutic strategy successful in one clinical scenario may not necessarily be effective in others. Experience from previous clinical trials suggests that this is the case. For example, distal embolisation protection devices have been shown to preserve microvascular function and reduce myocyte injury during SVG interventions but not in those undergoing primary PCI. ${ }^{6}$ In contrast, the use of glycoprotein IIb/IIIa antagonists in patients with acute coronary syndromes has been shown to preserve microvascular function, reduce periprocedural myocyte injury and improve clinical outcomes, while their use in patients undergoing SVG intervention has not been associated with significant benefit. ${ }^{3}$ Thus, while there are reasons to expect that vasodilators may be beneficial in treating no-reflow, their efficacy may vary in differing clinical situations and the optimal approach to preventing microvascular dysfunction will involve targeting multiple mechanisms.

\section{USE OF VASODILATORS IN NO-REFLOW: CURRENT KNOWLEDGE}

The periprocedural use of several different vasodilators for the prevention or treatment of no-reflow has been investigated in a number of observational studies and randomised trials ${ }^{17-14}$ These studies have varied widely in the patient group studied, methodology (drug, dose, route and frequency of administration) and the outcome measures used. Verapamil ${ }^{1-10}$ and adenosine $\mathrm{e}^{11-14}$ have been the vasodilators most frequently studied. Both these agents may improve myocardial perfusion by relieving small vessel spasm, but they also have additional effects which may reduce reperfusion injury. Verapamil has been shown to improve calcium

Abbreviations: AMISTAD, Acute Myocardial Infarction STudy of Adenosine; PCl, percutaneous coronary intervention; SVG, saphenous vein graff; TIMI,

Thrombolysis in Myocardial Infarction 
haemostasis in ischaemic myocardial cells, while adenosine has been shown to reduce neutrophil activation and endothelial injury. ${ }^{3}$

The studies investigating the use of verapamil to treat angiographic no-reflow following PCI to date have been small and have suggested that use of verapamil leads to an improvement in no-reflow as measured by TIMI flow rates and frame counts in the target vessel. ${ }^{17}$ Several small studies have also suggested that routine administration of intracoronary verapamil at the time of PCI prevents microvascular dysfunction following intervention in acute myocardial infarction or on saphenous vein grafts. ${ }^{8-10}$ Almost all the studies with adenosine to date have examined whether routine administration reduces no-reflow following PCI in the setting of acute myocardial infarction. Again several small studies have suggested that adenosine improves microvascular function and reduces infarct size. ${ }^{11-13}$ However, the AMISTAD II study, ${ }^{14}$ by far the largest study, enrolling 2118 patients with anterior ST elevation myocardial infarctions treated with either thrombolysis $(60 \%)$ or primary PCI $(40 \%)$, failed to demonstrate a reduction in the composite primary end point of death, new congestive heart failure or the first re-hospitalisation for congestive heart failure. There was a trend toward a reduction in the secondary end point of infarct size but this did not reach statistical significance.

In this issue of Heart Vijayalakshmi and colleagues ${ }^{15}$ report the results of a randomised comparison of intracoronary adenosine, verapamil or placebo in a mixed population containing both those with ST elevation and non-ST elevation acute coronary syndromes. This is the first trial to compare directly the effects of these vasodilators in an acute coronary syndrome population. Consistent with previous studies their results suggest that both verapamil and adenosine improved coronary blood flow in the target vessel and were associated with an improvement in wall motion index. Both drugs had similar benefits but verapamil was associated with hypotension and complete heart block lasting up to three hours in 18\% of the cases. The significance of these results should be interpreted with some caution. Although the improvements in TIMI frame counts and wall motion index were statistically significant, the size of the effect was relatively small and may not be of clinical significance.

\section{LIMITATIONS OF AVAILABLE STUDIES AND REMAINING QUESTIONS}

Although the study by Vijayalakshmi et $a l^{15}$ adds to our knowledge by providing further information about the comparative safety and efficacy of adenosine and verapamil, there remains a number of important unresolved issues. The optimal dose, route, frequency and duration of administration for these agents are unknown. For verapamil intracoronary administration of doses between $50-1000 \mu \mathrm{g}$ have been used. ${ }^{70}$ A dose of around $500 \mu \mathrm{g}$ has been the most commonly used and seems effective in improving microvascular flow but is associated with a risk of transient heart block. For adenosine intracoronary boluses between $24 \mu \mathrm{g}$ and $4 \mathrm{mg}$ have been used in some studies ${ }^{11}{ }^{12}$ while others have used intravenous infusions at rates of $10-70 \mu \mathrm{g} / \mathrm{kg} / \mathrm{min} .{ }^{13}{ }^{14}$ A subgroup analysis of the AMISTAD II trial $^{14}$ suggested that a higher infusion rate of $70 \mu \mathrm{g} / \mathrm{kg} / \mathrm{min}$ may be more effective. Unfortunately there are no studies comparing the relative efficacy of a prolonged intravenous infusion compared to a single or multiple intracoronary boluses.

It remains uncertain whether the use of vasodilators such as adenosine or verapamil to prevent or treat no-reflow reduces important clinical end points such as death or heart failure. As discussed previously the majority of studies have demonstrated improvements in a number of surrogate end points such as TIMI flow rate, TIMI frame count and wall motion scores. However, previous experience has taught us that there is frequently discordance between the results of trials using surrogate and clinical end points. This again proved to be the case with AMISTAD II, ${ }^{14}$ the only clinical trial adequately powered to demonstrate a difference in clinical outcomes failing to do so. Nevertheless, subgroup analysis did demonstrate a reduction in infarct size with the high dose adenosine infusion ( $70 \mu \mathrm{g} / \mathrm{kg} / \mathrm{min})$. This result was consistent with the findings of the AMISTAD I trial ${ }^{16}$ in which use of a $70 \mu \mathrm{g} / \mathrm{kg} / \mathrm{min}$ adenosine infusion in patients with ST elevation myocardial infarction treated with thrombolysis was also associated with a significant reduction in infarct size. Thus, it is possible that a $70 \mu \mathrm{g} / \mathrm{kg} / \mathrm{min}$ infusion may improve clinical outcomes, but this hypothesis needs to be confirmed by an adequately powered clinical trail.

\section{OPTIMAL TREATMENT TO REDUCE NO-REFLOW AND PERIPROCEDURAL MYOCYTE DAMAGE}

Given the complex and variable pathogenesis of no-reflow and periprocedural myocyte injury, multiple mechanisms need to be targeted with treatment being tailored to the clinical situation. Treatment with aspirin, clopidogrel, and statins before PCI reduce periprocedural myocyte damage and should be prescribed where possible. Use of glycoprotein IIb/IIIa antagonists in acute coronary syndrome interventions and distal embolisation protection devices in SVG interventions provide additional microvascular protection and improve clinical outcomes. It is difficult to recommend the routine use of adenosine or other vasodilators to prevent noreflow in acute coronary syndrome patients or other groups undergoing PCI based on the currently available evidence. Further large randomised controlled trials are required to resolve this issue. Should no-reflow occur following PCI, treatment with intracoronary adenosine or verapamil should be administered as this therapy is inexpensive, safe, improves flow in the target vessel and may reduce infarct size.

Conflict of interest statement: There are no conflicts of interest to declare.

\section{REFERENCES}

1 Piana R, Paik G, Moscucci $M$, et al. Incidence and treatment of 'no-reflow' after percutaneous coronary intervention. Circulation 1994;89:2514-8.

2 Abbo KM, Dooris M, Glazier S, et al. Features and outcome of no-reflow after percutaneous coronary intervention. Am J Cardiol 1995;75:778-82.

3 Eeckhout E, Kern MJ. The coronary no-reflow phenomenon: a review of mechanisms and therapies. Eur Heart J 2001;22:729-39.

4 Herrmann J. Peri-procedural myocardial injury: 2005 update. Eur Heart J 2005;26:2493-519.

5 Wilson RF, Laxson DD, Lesser JR, et al. Intense microvascular constriction after angioplasty of acute thrombotic coronary arterial lesions. Lancet 1989;i:807-1 1

6 Gick M, Jander N, Bestehorn H-P, et al. Randomized evaluation of the effects of filter-based distal protection on myocardial perfusion and infarct size after primary percutaneous catheter intervention in myocardial infarction with and without ST-segment elevation. Circulation 2005; 112:1462-9.

7 Werner GS, Lang K, Kuehnert $\mathrm{H}$, et al. Intracoronary verapamil for reversal of no-reflow during coronary angioplasty for acute myocardial infarction. Catheter Cardiovasc Interv 2002;57:444-51.

8 Taniyama $\mathrm{Y}$, Ito $\mathrm{H}$, Iwakura $\mathrm{K}$, et al. Beneficial effect of intracoronary verapamil on microvascular and myocardial salvage in patients with acute myocardial infarction. J Am Coll Cardiol 1997;30:1193-9.

9 Michaels AD, Appleby M, Otten MH, et al. Pretreatment with intragraft verapamil prior to percutaneous coronary intervention of saphenous vein graft lesions: results of the randomized, controlled vasodilator prevention on noreflow (VAPOR) trial. J Invasive Cardiol 2002;14:299-302.

10 Hang C-L, Wang C-P, Yip H-K, et al. Early administration of intracoronary verapamil improves myocardial perfusion during percutaneous coronary interventions for acute myocardial infarction. Chest 2005;128:2593-8.

11 Assali AR, Sdringola S, Ghani M, et al. Intracoronary adenosine administered during percutaneous intervention in acute myocardial infarction and reduction in the incidence of "no reflow" phenomenon. Catheter Cardiovasc Interv 2000;51:27-31.

12 Marzilli M, Orsini E, Marraccini P, et al. Beneficial effects of intracoronary adenosine as an adjunct to primary angioplasty in acute myocardial infarction. Circulation 2000;101:2154-9.

13 Micari A, Belcik TA, Balcells EA, et al. Improvement in microvascular reflow and reduction of infarct size with adenosine in patients undergoing primary coronary stenting. Am J Cardiol 2005;96:1410-5. 
14 Ross AM, Gibbons RJ, Stone GW, et al. A randomized, double-blinded, placebo-controlled multicenter trial of adenosine as an adjunct to reperfusion in the treatment of acute myocardial infarction (AMISTAD-II). J Am Coll Cardiol 2005:45:1775-80.

15 Vijayalakshmi K, Whittaker VJ, Kunadian B, et al. Prospective, randomised controlled trial to study the effect of intracoronary injection of verapamil and adenosine on coronary blood flow during percutaneous coronary intervention in patients with acute coronary syndromes. Heart 2006;92:1278-84.

16 Mahaffey KW, Puma JA, Barbagelata NA, et al. Adenosine as an adjunct to thrombolytic therapy for acute myocardial infarction: results of a multicenter, randomized, placebo-controlled trial: the acute myocardial infarction study of adenosine (AMISTAD) trial. J Am Coll Cardiol 1999;34:1711-20.

\section{IMAGES IN CARDIOLOGY}

doi: 10.1136/hrt.2005.084194

Anomalous origin of the left main coronary artery from the main pulmonary artery (ALCAPA) illustrated before and after surgical correction on ECG-gated 40-slice computed tomography

A 39-year-old man was admitted in another institution for diagnostic work-up of stable exercise induced angina pectoris. Clinical examination was unremarkable. Exercise SestaMIBI single photon emission computed tomography (SPECT) revealed a large perfusion defect in the anterior territory, normalising at rest (panel A). Cardiac catheterisation revealed a large right coronary artery (panel B) in orthotopic location. However, catheterisation of the left main coronary artery (LMCA) was not possible. Therefore the patient was referred to our institution for multislice computed tomography (CT), to document the location and origin of the LMCA.

Enhanced multi-slice CT (MX Brilliance 40, Philips Medical Systems, Cleveland, Ohio, USA) was performed with retrospective ECG-gating, $40 \times 0.625 \mathrm{~mm}$ collimation, $120 \mathrm{kV}$ and 600 mAs. Globe view reconstructed CT images revealed a large right coronary artery arising from the aorta (Ao) (panel C, straight arrow) and an aberrant LMCA (curved arrow) originating from the main pulmonary artery (PA). A retrospective review of the catheter lab images showed the LMCA perfused by collaterals developed from the right coronary artery (panel D, curved arrow) and directly connected to the pulmonary artery. The patient underwent surgical reimplantation of the LMCA on the ascending aorta. A follow-up enhanced multi-slice CT demonstrated the patency of the reimplanted LMCA (panel E) (straight arrow) in the left coronary sinus (AO).

To our knowledge this is the first reported case of anomalous origin of the left main coronary artery from the main pulmonary artery (ALCAPA) documented before and after surgery correction in an adult patient.

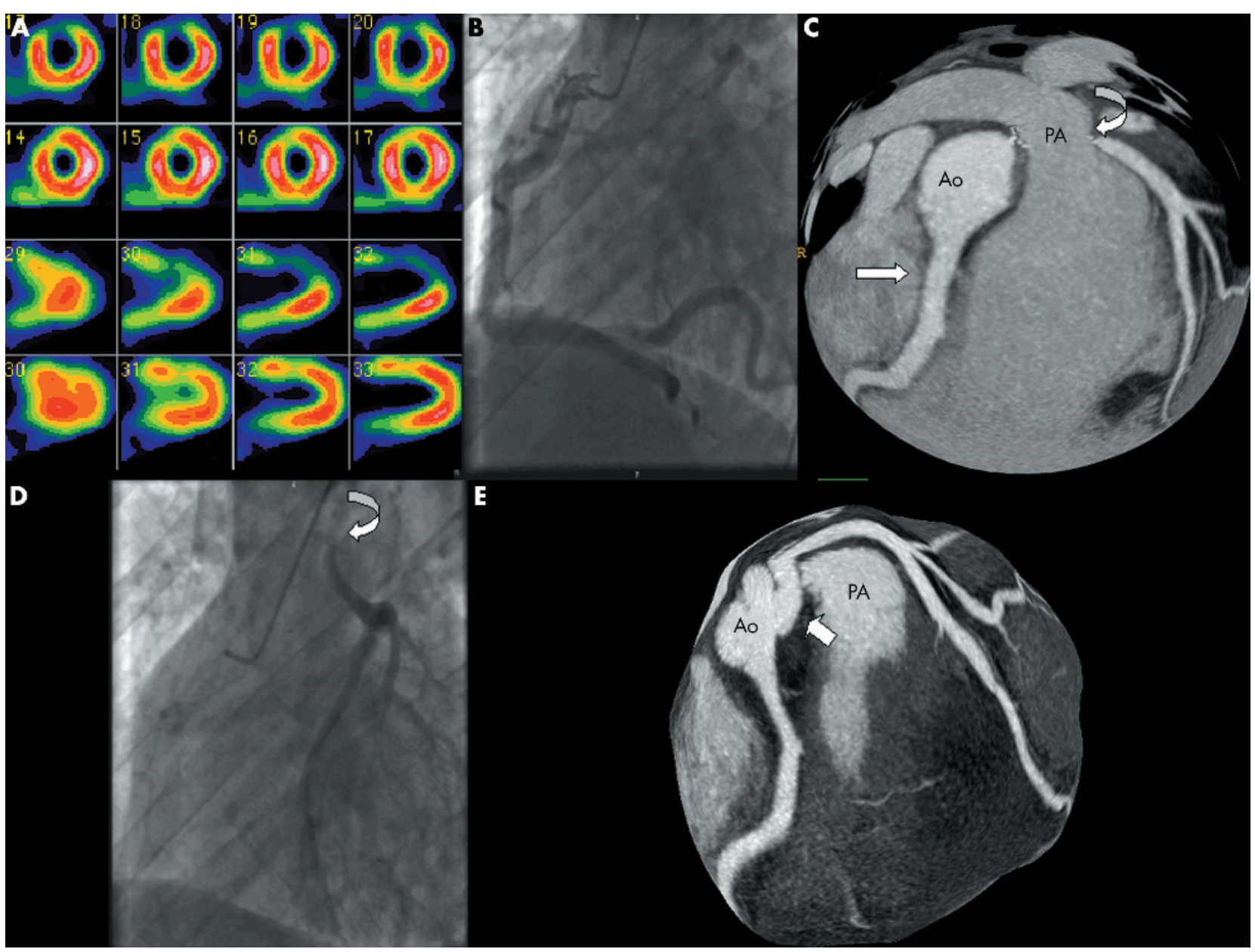

